On the other hand, it is possible that the histone modifications are not restored. Instead, the presence of new histones could form a memory of the damage. This could serve an adaptive role in the recovery process or, instead, be maladaptive, and help to explain the occurrence of radiationinduced genomic instability in the progeny of the damaged cells.

More mechanistic studies are now needed to distinguish between these possibilities and explain how epigenetics interacts with damage repair. The experiments also need to be extended to other histone variants to test their generality.

ORIGINAL RESEARCH PAPER Polo, S. E. Roche, D. \& Almouzni, G. New histone incorporation marks sites of UV repair in human cells. Cell 127, 481-493 (2006)

ORIGINAL RESEARCH PAPER Varrault, A. et al. Zac1 regulates an imprinted gene network critically involved in the control of embryonic growth. Dev. Cell 11, 711-722 (2006) FURTHER READING Reik, W. \& Lewis, A. Co-evolution of X-chromosome inactivation and imprinting in mammals. Nature Rev. Genet. 6 , 403-410 (2005) | Barabasi, A.-L. \& Oltvai, Z. N. Network biology: understanding the cell's functional organization. Nature Rev. Genet. 5 , 101-113 (2004)

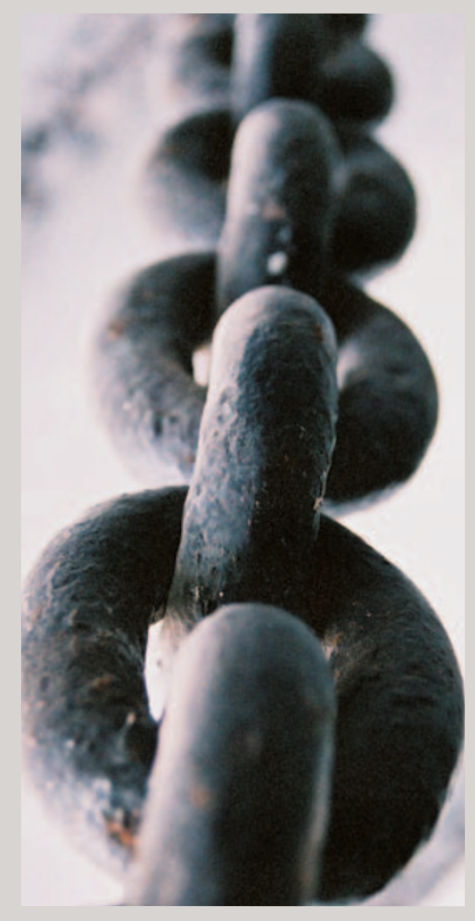

Image courtesy of D. Jones.

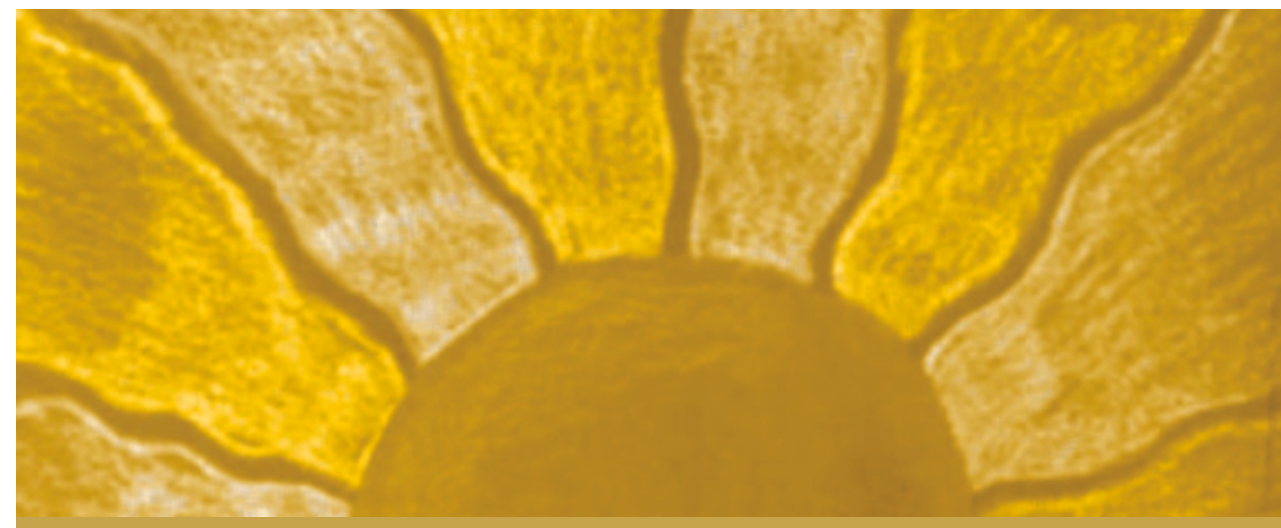

GENOMICS

\title{
The dawn of Neanderthal genomics
}

Initial sequencing and analysis of Neanderthal genomic DNA have recently been reported. Neanderthals, who became extinct some 30,000 years ago, are our closest hominid relatives; their genome therefore offers unique opportunities to identify the genetic changes that are specific to modern humans, facilitating our understanding of both human and other hominid biology and evolution.

A group led by Eddy Rubin used metagenomics - an approach previously developed for environmental genomics and for ancient DNA cloning - combined with Sanger sequencing and massive parallel pyrosequencing to obtain $\sim 65,000$ bp of Neanderthal genome sequence. At the same time, Svante Pääbo and colleagues used direct high-throughput sequencing to obtain $\sim 752,000$ bp of unique Neanderthal nuclear DNA. Both groups used a Neanderthal bone sample from Croatia - a male, as it turns out, as both groups detected Y-chromosome sequences in their reads.

Sequencing DNA from ancient samples is fraught with problems such as contamination with DNA from other sources; for example, from modern humans and organisms that have colonized the ancient remains. Between them, the groups used a number of methods to ensure that what they were analysing was genuine Neanderthal DNA: comparisons of PCRamplified mitochondrial DNA sequences from the ancient sample and modern humans, analysis of signatures of DNA damage that are characteristic of ancient DNA (deamination of cytosine to uracil is frequently found in ancient DNA) and sequence comparisons to human and chimpanzee orthologous sequences.

Comparison with chimpanzee sequences also allowed the authors to identify the number of mutations that are specific to each of these lineages. For example, Green et al. estimate that $\sim 8 \%$ of the DNA sequence changes in the human lineage have occurred after divergence from the Neanderthal.

Given the current human-chimpanzee divergence, Noonan et al. estimate that, on average, modern human and Neanderthal genomes shared a most recent common ancestor $\sim 706,000$ years ago, and that the human and Neanderthal ancestral populations split $\sim 370,000$ yrs ago. Green et al. put humanNeanderthal DNA sequence divergence at $\sim 516,000$ yrs.

As late as 30,000 years ago, modern humans and Neanderthals co-existed in Europe. Although there is no archaeological evidence that they lived together, the nature of their interaction and, most intriguingly, the question of whether any interbreeding between the two species took place, are subject to debate. By comparing the frequency of the ancestral and derived alleles in Neanderthals and humans, both groups looked at admixture between the two. Although Noonan et al. find little evidence for its existence, Green et al. suggest that some gene flow from humans (in particular from human males) to Neanderthals might have occurred.

Ultimately, a definitive answer to this and other questions will require additional sequence data. And more data will be available - given the advances in sequencing technologies and procedures for retrieval of DNA from ancient samples, a project to produce a draft version of the Neanderthal genome has been initiated.

The authors promise the draft sequence within two years.

Magdalena Skipper

ORIGINAL RESEARCH PAPERS Noonan, J. P. et al. Sequencing and analysis of Neanderthal genomic DNA. Science 314, 1113-1118 (2006) | Green, R. E. et al. Analysis of one million base pairs of Neanderthal DNA. Nature 444, 330-336 (2006) FUTHER READING Tringe, S. G. \& Rubin, E. M. Metagenomics: DNA sequencing of environmental samples. Nature Rev. Genet. 6, 805-814 (2005) 